\title{
Effect of revascularization on IL-6 and TNF- $\alpha$ in patients with thromboangiitis obliterans
}

\author{
SHIFENG WU ${ }^{1}, \mathrm{XIN} \mathrm{SUN}^{2}$, WEI WU ${ }^{3}, \mathrm{DAZHI} \mathrm{SHI}^{4}$ and TAO JIANG ${ }^{5}$ \\ ${ }^{1}$ Department of Burns and Plastic Surgery, China-Japan Union Hospital of Jilin University; \\ Departments of ${ }^{2}$ Neurology and ${ }^{3}$ Neurosurgery, The First Hospital of Jilin University; \\ ${ }^{4}$ Department of Intensive Care Unit, The Fourth Hospital of Jilin University; ${ }^{5}$ Department of Vascular \\ Surgery, China-Japan Union Hospital of Jilin University, Changchun, Jilin 130033, P.R. China
}

Received August 29, 2017; Accepted February 1, 2018

DOI: $10.3892 /$ etm.2018.5877

\begin{abstract}
The aim of the study was to investigate the effects of revascularization in treating patients with thromboangiitis obliterans (TAO), to analyze the prognosis of TAO. The treatment group comprised 32 patients with TAO of lower limbs who were selected between March 2012 and March 2017. Patients in the treatment group were treated with revascularization (vascular bypass surgery, catheter-directed thrombolysis and angioplasty, endovascular angioplasty + stening, thromboectomy and/or endarterectomy) + Western medicine. Another 33 patients with TAO who were treated with Western medicine alone comprised the control group. Treatment outcomes were compared between the groups. Serum levels of interleukin-6 (IL-6), interleukin-8 (IL-8) and tumor necrosis factor- $\alpha$ (TNF- $\alpha$ ) were also detected and compared between the groups. Multivariate analysis was performed to identify the factors related to prognosis. Compared with control group, treatment outcomes were significantly better in the treatment group $(\mathrm{P}<0.05)$. After treatment, the serum levels of IL- 6 , IL-8 and TNF- $\alpha$ significantly decreased in both groups, and the decrease in the treatment group was more significant $(\mathrm{P}<0.01)$. Multivariate analysis revealed that vascular bypass surgery and preoperative ischemic degree are associated with treatment effect. Our results show that revascularization treatment of TAO is conducive to clinical symptoms and dysfunction of inflammatory cytokines, and the type of surgery and surgical timing significantly affect treatment outcomes.
\end{abstract}

\section{Introduction}

Thromboangiitis obliterans (TAO) is a progressive, segmental, and non-infectious inflammatory disease. TAO is a small and

Correspondence to: Dr Tao Jiang, Department of Vascular Surgery, China-Japan Union Hospital of Jilin University, 128 Xiantai Street, Changchun, Jilin 130033, P.R. China

E-mail:tvdlva@163.com

Key words: thromboangiitis obliterans, revascularization, IL-6, IL- 8, TNF- $\alpha$ middle artery occlusion caused by inflammation of the whole vascular wall, often affecting the adjacent veins and nerves. TAO is more common in young men (1). The pathological mechanism is mainly the secondary vascular inflammatory cell infiltration in diseased blood vessels, thrombosis, luminal occlusion, and limb (more common in lower extremity) ischemic changes (2).

The early manifestation of TAO is mainly intermittent claudication, and late manifestations include rest pain, acral ulcers and even gangrene. High amputation may be needed for severe cases (3), seriously affecting the life and health of the patient. At present, there are no effective treatments for TAO. Without outflow tract, most scholars do not support the use of direct reconstruction in the treatment of TAO (4). Inflammation is closely associated with the development of TAO.

In the present study, 32 patients with TAO were selected between March 2012 and March 2017 to be treated with revascularization and Western medicine, and a further 33 patients with TAO who were treated solely with Western medicine served as the control group. The effects of reconstruction on IL-6, IL-8 and TNF- $\alpha$ in patients with TAO were explored to guide the treatment and prognosis of TAO.

\section{Patients and methods}

Patients. In total, 65 male patients diagnosed with TAO selected between March 2012 and March 2017 in China-Japan Union Hospital of Jilin University Hospital (Changchun, China) were included in the present study. There were 32 patients in the treatment group, with a mean age of $39.38 \pm 7.45$ years, the average duration of disease was $2.65 \pm 0.58$ years and the average BMI $\left(24.21 \pm 4.35 \mathrm{~kg} / \mathrm{m}^{2}\right)$. There were 23 cases of ischemic period, 8 cases of nutritional disorder and 1 case of gangrene, and the main symptoms included intermittent claudication in 23 cases and rest pain in 9 cases. There were 33 patients in the control group, with a mean age of $39.21 \pm 7.38$ years, the average course of disease $2.73 \pm 0.47$ years and the average BMI $\left(24.34 \pm 3.93 \mathrm{~kg} / \mathrm{m}^{2}\right)$. There were 24 cases of ischemic period, 8 cases of nutritional disorders and 1 case of gangrene, and the main symptoms included intermittent claudication in 24 cases and rest pain in 9 cases. No significant differences in general clinical data were found between the two groups $(\mathrm{P}>0.05)$. The general data of patients are shown in Table I. 
Table I. Comparison of general clinical data between two groups of patients.

\begin{tabular}{lcccr}
\hline General information & $\begin{array}{c}\text { Treatment group } \\
(\mathrm{n}=32)\end{array}$ & $\begin{array}{c}\text { Control group } \\
(\mathrm{n}=33)\end{array}$ & $\mathrm{t} / \chi^{2}$ value & P-value \\
\hline Age (years) & $39.18 \pm 7.45$ & $39.21 \pm 7.38$ & 1.729 & 0.168 \\
Course of illness & $2.65 \pm 0.58$ & $2.73 \pm 0.47$ & 1.867 & 0.115 \\
BMI $\left(\mathrm{kg} / \mathrm{m}^{2}\right)$ & $24.21 \pm 4.35$ & $24.34 \pm 3.93$ & 1.645 & 0.186 \\
Stages $[\mathrm{n}(\%)]$ & & & & 0.089 \\
Local ischemia & 23 & 24 & & 0.094 \\
Nutritional disorder & 8 & 8 & 1.981 & \\
Gangrene & 1 & & & \\
Main symptoms [n $(\%)]$ & 23 & 24 & & \\
Intermittent claudication & 9 & 9 & & \\
Rest pain & & & &
\end{tabular}

The study was approved by the Ethics Committee of the China-Japan Union Hospital of Jilin University. Informed consents were signed by the patients and/or guardians.

Inclusion and exclusion criteria. Patients were diagnosed according to Highlander et al (5). The majority of patients were male, approximately 20-40 years of age. Primary symptoms included angina cruris; resting pain, soreness, swelling and numbness (show 1 or more symptoms); cold or burning sensation (show at least 1 symptom); dystrophic change in skin, hair, muscles, toe (finger); toes (or/and foot) gangrene or finger gangrene (mostly dry gangrene); and recurrent thrombophlebitis of the leg or foot. The fluctuations of middle artery and/or of the affected limb decreased or disappeared. Tongue demonstration involved whether the tongue was rare lavender, purple, indicating petechiae or ecchymosis. Pulse condition was classified as wiry and tense; deep and unsmooth; or weak pulse. Diagnostic criteria included $1+3+4+a, b$, a and $b+$ one of the symptoms left in the primary symptoms.

Exclusion of arteriosclerosis obliterans. Exclusion criteria for the study were: vascular thrombosis; rheumatism, autoimmune diseases (Takayasu syndrome, psoriasis and Raynaud's disease); diabetes or other ischemic chronic nutritional disorders caused by local damage.

Treatment. Patients in the control group were treated with Western medicine alone, while patients in the control group were treated with revascularization + Western medicine to improve blood circulation. The course of treatment was 3 months.

Western medicine treatment. The Western medicine consisted of $10 \mathrm{mg}$ of hydrochloric acid nicardipine sustained-release tablets (Chongqing Kerui Pharmaceutical Group Co., Ltd., SFDA approval no.: H10980159) + $10 \mathrm{mg}$ of enteric-coated aspirin (Shuguang Pharmaceutical Co., Ltd., SFDA approval no.: H11020827) + enteric-coated trental (Datong Weihua Pharmaceutical Co., Ltd., SFDA approval no.: 0421106). The three drugs were taken orally once daily. Analgesic treatment was performed for patients with severe pain.
Table II. Comparison of treatment outcomes between two groups.

\begin{tabular}{lcccc}
\hline Efficacy & $\begin{array}{c}\text { Treatment } \\
\text { group } \\
(\mathrm{n}=32)\end{array}$ & $\begin{array}{c}\text { Control } \\
\text { group } \\
(\mathrm{n}=33)\end{array}$ & $\chi^{2}$ value & P-value \\
\hline Cure & 26 & 21 & & \\
Markedly effective & 2 & 3 & 3.265 & 0.038 \\
Effective & 2 & 3 & & \\
Ineffective & 2 & 6 & 6.743 & 0.002 \\
Limb salvage rate & $90.63 \%$ & $81.82 \%$ & 4.125 & 0.023 \\
\hline
\end{tabular}

Revascularization. According to the characteristics of local vascular lesions, artery intervention revascularization (catheter-directed thrombolysis and angioplasty, endovascular angioplasty + stening, thromboectomy and/or endarterectomy) or vascular bypass surgery was used. Vascular bypass surgery (large saphenous vein, artificial blood vessels, composite bridge blood vessels of artificial blood vessels and large saphenous vein) was performed in 11 cases, accounting for $34.375 \%$; catheter-directed thrombolysis and angioplasty was performed for 9 cases, accounting for $28.125 \%$; endovascular angioplasty + stening was performed for 7 cases, accounting for $21.875 \%$; thromboectomy + endarterectomy was performed for 5 cases, accounting for $15.625 \%$.

Detection method. Fasting elbow venous blood (3-5 ml) was extracted in the morning before and at 3 months after treatment. Blood was centrifuged at $8,000 \mathrm{x}$ g for $15 \mathrm{~min}$ to collect serum. IL- 6 and IL- 8 were detected by radioimmunoassay. TNF- $\alpha$ was detected by double antibody sandwich ELISA.

Criteria for the determination of efficacy. Efficacy was determined using the 6 levels method, as previously described (5). Scores were recorded as ' $0-5$ ', and the higher score indicate severe condition. Scores before and after treatment were compared: 0 points, a cure, 2 points drop represents markedly effective, and 
Table III. Changes in inflammatory cytokines in the two groups (ng/l, mean \pm SD).

\begin{tabular}{lccccc}
\hline & \multicolumn{2}{c}{ Treatment group $(\mathrm{n}=32)$} & & \multicolumn{2}{c}{ Control group $(\mathrm{n}=33)$} \\
\cline { 2 - 3 } $\begin{array}{l}\text { Observation } \\
\text { indicators }\end{array}$ & Before treatment & After treatment & & Before treatment & After treatment \\
\hline IL-6 & $4.32 \pm 2.30$ & $1.24 \pm 2.35^{\mathrm{a}, \mathrm{b}}$ & $4.28 \pm 2.34$ & $2.19 \pm 2.27^{\mathrm{a}}$ \\
IL-8 & $843.21 \pm 95.32$ & $250.76 \pm 78.12^{\mathrm{a}, \mathrm{b}}$ & & $646.15 \pm 92.56$ & $3.05 \pm 1.60^{\mathrm{a}}$ \\
TNF- $\alpha$ & $4.57 \pm 2.12$ & $1.64 \pm 1.53^{\mathrm{a}, \mathrm{b}}$ & $4.55 \pm 2.14$ & $6.33^{\mathrm{a}}$ \\
\hline
\end{tabular}

${ }^{\text {a }} \mathrm{P}<0.05$, compared with pre-treatment level; ${ }^{\mathrm{b}} \mathrm{P}<0.05$ compared with control group.

Table IV. Regression analysis and the relationship between various factors and prognosis.

\begin{tabular}{lccccc}
\hline Variables & $\begin{array}{c}\text { Regression } \\
\text { coefficients }\end{array}$ & $\begin{array}{c}\text { Standard } \\
\text { error }\end{array}$ & Wald value & $\begin{array}{c}\text { Degrees of } \\
\text { freedom }\end{array}$ & P-value \\
\hline Treatment methods & 3.023 & 1.521 & 4.073 & 1 & 0.032 \\
Smoking & 0.948 & 1.398 & 0.434 & 1 & 0.481 \\
Age & 0.385 & 1.278 & 0.089 & 1 & 0.716 \\
Degree of ischemia & -3.102 & 1.532 & 4.121 & 1 & 0.035 \\
\hline
\end{tabular}

1 point drop represents effective. Unchanged scores represent increased severity or ineffective treatment. Cure, markedly effective and effective results were recorded as improvement.

Statistical analysis. SPSS 20.0 software (SPSS Inc., Chicago, IL, USA) software was used for all statistical analysis. Enumeration data were expressed as cases or ratio, and were processed by Fisher's exact test or Chi-square test. Normal distribution measurement data were expressed as (mean \pm SD), and were processed using paired sample t-test. Abnormal distribution measurement data were processed using the Wilcoxon two-sample test. $\mathrm{P}<0.05$ was considered to indicate a statistically significant difference.

\section{Results}

Comparison of treatment outcomes between the groups. In treatment group, 26 patients were cured, markedly effective results were found in 2 cases, effective results were found in 2 cases, and ineffective results in 2 cases ( 1 case was treated with vascular bypass, 3 cases were subjected to amputation), and the limb salvage rate was $90.63 \%$ (29/32). In control group, 21 patients were cured, markedly effective results were found in 3 cases, effective results were found in 3 cases and ineffective results in 6 cases ( 1 case was treated with vascular bypass, 6 cases were subjected to amputation), and the limb salvage rate was $81.82 \%$ (27/33). The overall limb salvage rate was $86.15 \%$ (56 cases/65 cases). Compared with the control group, treatment outcomes were significantly improved and the limb salvage rate was significantly increased in the treatment group $(\mathrm{P}<0.05)$ (Table II).

Changes in inflammatory cytokines in the groups. Before treatment, no significant differences in the serum levels of inflammatory cytokines were found between the groups $(\mathrm{P}>0.05)$. Compared with the pre-treatment levels, the serum levels of IL-6, IL-8 and TNF- $\alpha$ were significantly reduced in both groups, and the decreases of serum levels of IL-6, IL-8 and TNF- $\alpha$ were more significant in the treatment group (Table III).

Single factor regression analysis. Patients were divided into the improvement and ineffective groups. The improvement group (cure, markedly effective and effective) included 57 patients, and the ineffective group included 8 cases. Age, BMI, duration of disease, smoking, disease stages (local ischemic period, nutritional disorders, gangrene), main symptoms (intermittent lame and rest pain), preoperative ischemic degree (with or without ulcers), and treatment method (with or without vascular bypass surgery) were compared between the two groups. Significant differences in smoking, age and degree of ischemia were found $(\mathrm{P}<0.05)$. No significant differences in $\mathrm{BMI}$, disease stages (local ischemic period, nutritional disorders, gangrene) or main symptoms (intermittent lame and rest pain) were identified between the groups.

Multi-factor regression analysis. Age, BMI, duration of disease, smoking, disease stages, main symptoms (intermittent lame and rest pain), preoperative ischemic degree (with or without ulcers), and treatment method (with or without vascular bypass surgery) were subjected to correlation analysis. However, no significant correlations were identified among the factors. Independent variables with significant differences in univariate analysis were subjected to multivariate analysis of logistic regression model. Results showed that vascular bypass surgery, preoperative ischemia and treatment method were closely correlated with treatment outcomes (Table IV).

\section{Discussion}

Sensory disturbance caused by limb ischemia is common in patients with Thromboangiitisobliterans (TAO).Approximately 
$50-80 \%$ of TAO patients showed rest pain or ischemic ulcer (6) and even local nutritional disorders. Without timely treatment, necrosis (7), organization (8) and gangrene (9) caused by ischemia and hypoxia may occur. Cell metabolism is disturbed in necrotic limbs, and harmful substances are also released to threaten patients' life. Amputation is needed in some cases to save life, leading to high morbidity. Thus, identifying the cause and creating an effective treatment plan is the focus of the treatment of TAO. No effective treatments are available for $\mathrm{TAO}$, and the application of reconstruction in TAO treatment has been controversial (4). Indications of revascularization should be clarified prior to treatment and only patients with good outflow tract can be treated with revascularization (10). In this study, in the treatment group, 26 patients were cured, 2 cases were markedly improved, 2 cases were effectively improved, and ineffective results were found in 2 cases ( 1 case was treated with vascular bypass, 3 cases were subjected to amputation), and the limb salvage rate was $90.63 \%$ (29/32). In the control group, 21 patients were cured, 3 cases were markedly improved, 3 cases were improved and ineffective results were found in 6 cases ( 1 case was treated with vascular bypass, 6 cases were subjected to amputation), and the limb salvage rate was $81.82 \%$ (27/33). Amputation rate in the treatment group was $9.375 \%$ ( 3 cases $/ 32$ cases), and 3 patients received secondary surgery. Therefore, patients with poor conditions cannot be treated with this treatment, and amputation should be performed to improve the quality of life of patients with the use of prosthetics.

In the present study, multivariate analysis revealed that vascular bypass and treatment efficacy were related; therefore, autologous vein surgery was used more commonly. Vascular bypass surgery has some restrictions in the clinical practice (11). First, the selection of outflow tract means TAO can affect distal knee artery. Thus, anterior tibial artery, posterior artery or peroneal artery is generally used as the outflow tract. However, the arterial anatomical locations of those arteries are deep and surrounding nerves and veins can be easily damaged during operation. Therefore, only experienced surgeons can perform this operation. Furthermore, regarding the supply of vascular blood vessels, the preferred bridge blood vessel material is a large autologous vein. TAO patients are often accompanied by superficial venous thrombosis. It is generally believed that large saphenous vein with a diameter of $>4 \mathrm{~mm}$ is good material. Artificial blood vessels and large saphenous vein composite material can be used as an alternative if large saphenous vein cannot be obtained. In addition, with regard to distal arterial anastomosis, a microscope should be used for operations on peroneal artery. Thrombolysis and angioplasty can be used when autologous venous bridge blood vessels cannot be obtained. In TAO patients showing both distal arterial chronic occlusion and proximal thrombosis, simple thrombolytic therapy may be enough to achieve good efficacy, while thrombolytic therapy combined with distal arterial dilatation may be used to achieve improved results. However, thromboectomy and/or endarterectomy may not be enough, and these two treatments should be avoided for young patients (12). Smoking is, not only a cause of TAO, but also a risk factor of poor prognosis (13). The pathogenesis of TAO is unclear, but smoking, low temperature, immune response and other factors may be involved (14-16). Although the present study did not suggest the relevance of smoking and end results, all 9 cases of amputation were smoker. Thus, this small sample size may still be sufficient to highlight the importance of quiting smoking.

The pathology of TAO is inflammatory cell infiltration caused by vascular inflammation (17). Therefore, some scholars believe that TAO is a form of autoimmune inflammatory disease (18). Vascular endothelial cell damage is an important aspect of the occurrence of TAO (19). Through endocrine function and paracrine synthesis, vascular endothelial cells release a considerable amount of vasodilator and vasoconstrictor substances to regulate and protect the vascular structure and functional integrity. Once vascular endothelium is damaged, the endothelial anticoag ulation, anti-platelet and anti-fibrinolytic function, vasomotor and secretion and other functions may not be maintained. Abnormal secretion of cytokines can promote platelet aggregation and inflammatory response by altering endothelial permeability to cause endothelial structural damage (20). As the central mediators of immune and inflammatory responses, IL-6 and IL-8 are important pro-inflammatory cytokines produced by macrophages (21). TNF- $\alpha$ is a factor with direct cytotoxicity produced by activated monocyte macrophages. TNF- $\alpha$ can promote oxidative metabolism, accelerate lipid peroxidation process, and destroy vascular endothelial cell structure integrity through neutrophil degranulation to cause dysfunction, promote IL-6 and IL-8 secretion and regulate vascular smooth muscle cell differentiation, eventually leading to wall thickening, stenosis and increased blood flow resistance (22). Previous findings have shown that TNF- $\alpha$ can be used as an important indicator of vascular endothelial dysfunction (23). Immune regulation dysfunction and cell physiological dysfunction in TAO patients can affect the immune system to cause clinical symptoms (24).

In the present study, the high levels of IL-6, IL-8 and TNF- $\alpha$ indicated the existing of cytokine metabolic disorders. After treatment, different degrees of decrease in those cytokine were observed in both groups, suggesting that clinical treatment can improve metabolic disorders of cytokines in patients. After treatment, the levels of cytokines were found to be significantly lower in the treatment group than in the control group $(\mathrm{P}<0.05)$, suggesting that the treatment of TAO with revascularization can better improve limb blood supply and immune injury. Results of the current study have shown that revascularization is a safe and effective method than can significantly improve the symptoms of patients. The identification of surgical indications and proper perioperative management can improve treatment efficacy, reduce inflammatory response, and improve immune dysfunction, so as to provide a good basis for follow-up rehabilitation.

\section{Acknowledgements}

Not applicable.

\section{Funding}

No funding was received.

\section{Availability of data and materials}

The datasets used and/or analyzed during the current study are available from the corresponding author on reasonable request. 


\section{Authors' contributions}

SW contributed to the conception and design of the study, and approved the final manuscript. XS and TJ collected and reviewed the data and was resonsible for the follow-up management of the patients. WW and DS were responsible for the statistical analysis of the data. TJ drafted and revised the manuscript critically for important intellectual content. All authors read and approved the final manuscript.

\section{Ethics approval and consent to participate}

The study was approved by the Ethics Committee of China-Japan Union Hospital of Jilin University (Changchun, China). Informed consents were signed by the patients and/or guardians.

\section{Consent for publication}

Not applicable.

\section{Competing interests}

The authors declare that they have no competing interests.

\section{References}

1. Mills JL Sr: Buerger's disease in the 21st century: Diagnosis, clinical features, and therapy. Semin Vasc Surg 16: 179-189, 2003.

2. Kocak B and Ustun ED: Vasa nervorum as the source of corkscrew collaterals in thromboangiitis obliterans (Buerger's Disease). Eur J Vasc Endovasc Surg 52: 508, 2016.

3. Klein-Weigel PF and Richter JG: Thromboangiitis obliterans (Buerger's disease). Vasa 43: 337-346, 2014.

4. Rivera-Chavarría IJ and Brenes-Gutiérrez JD: Thromboangiitis obliterans (Buerger's disease). Ann Med Surg (Lond) 7: 79-82, 2016.

5. Highlander P, Southerland CC, VonHerbulis E and Gonzalez A:Buerger disease (thromboangiitis obliterans): A clinical diagnosis. Adv Skin Wound Care 24: 15-17, 2011.

6. Szuba A and Cooke JP: Thromboangiitis obliterans. An update on Buerger's disease. West J Med 168: 255-260, 1998

7. Rimar D, Rozenbaum M, Slobodin G, Boulman N, KaLy L and Rosner I: Thromboangiitis obliterans overlapping with atherosclerotic occlusive arterial disease: Small merenteric artery involvement by thromboangiitis obliterans. Harefuah 155 : 41-44,67,66, 2016 (In Hebrew)

8. Abyshov NS, Abdullayev AG, Zakirdzhaev ED, Guliyev RA, Akhmedov MB, Tagizade GT, Zeynalova GM and Mamedova LD The results of combined surgical treatment of thromboangiitis obliterans and critical lower limb ischemia using prolonged epidural analgesia and autohemotherapy with ozone. Khirurgiia (Mosk) 9: 45-50, 2016 (In Russian).
9. Aszalos C, Dongó E, Farkas Z, Kollár A, Magyar P, Várallyay G Bereczki D and Vastagh I: Cerebral manifestations of thromboangiitis obliterans. Case report. Orv Hetil 157: 1207-1211, 2016 (In Hungarian)

10. Kawarada O, Sakamoto S, Harada K, Ishihara M, Yasuda S and Ogawa H: Endovascular recanalization of failed distal bypass in Buerger's disease. Cardiovasc Interv Ther 29: 266-269, 2014.

11. Zeng R, Ye W, Li YJ, Shao J, Zheng YH and Liu CW: Revascularization for lower limbs thromboangiitis obliteransearly and mid-term outcome report. Zhonghua Wai Ke Za Zhi 51: 719-722, 2013 (In Chinese)

12. Shionoya S, Ban I, Nakata Y, Matsubara J, Hirai M and Kawai S: Surgical treatment of Buerger's disease. J Cardiovasc Surg (Torino) 21: 77-84, 1980.

13. Piazza G and Creager MA: Thromboangiitis obliterans. Circulation 121: 1858-1861, 2010.

14. Owlia MB and Mehrpoor G: Thromboangiitis obliterans with cryoglobulinemia. J Coll Physicians Surg Pak 24: 863-864, 2014

15. Modaghegh MH, Kazemzadeh GH, Ravari H, Johari HG and Barzanuni A: Buerger's disease in the northeast of Iran: Epidemiology and clinical features. Vascular 23: 519-524, 2015.

16. Sapkota P, Budhathoky $P$ and Mathew S: Smoking and Thromboangitis Obliterans - Are they related? JNMA J Nepal Med Assoc 52: 802-805, 2014.

17. Johnson JA and Enzenauer RJ: Inflammatory arthritis associated with thromboangiitis obliterans. J Clin Rheumatol 9: 37-40, 2003.

18. Puéchal X and Fiessinger JN: Thromboangiitis obliterans or Buerger's disease: Challenges for the rheumatologist. Rheumatology (Oxford) 46: 192-199, 2007.

19. Azizi M, Boutouyrie P, Bura-Rivière A, Peyrard S, Laurent S and Fiessinger JN: Thromboangiitis obliterans and endothelial function. Eur J Clin Invest 40: 518-526, 2010.

20. Hartge MM, Kintscher U and Unger T: Endothelial dysfunction and its role in diabetic vascular disease. Endocrinol Metab Clin North Am 35: 551-560, 2006.

21. Slavov ES, Stanilova SA, Petkov DP and Dobreva ZG: Cytokine production in thromboangiitis obliterans patients: New evidence for an immune-mediated inflammatory disorder. Clin Exp Rheumatol 23: 219-226, 2005.

22. Halacheva K, Gulubova MV, Manolova I and Petkov D: Expression of ICAM-1, VCAM-1, E-selectin and TNF-alpha on the endothelium of femoral and iliac arteries in thromboangiitis obliterans. Acta Histochem 104: 177-184, 2002.

23. Huang WY, Wang J, Liu YM, Zheng QS and Li CY: Inhibitory effect of Malvidin on TNF- $\alpha$-induced inflammatory response in endothelial cells. Eur J Pharmacol 723: 67-72, 2014.

24. Dellalibera-Joviliano R, Joviliano EE, Silva JS and Evora PR: Activation of cytokines corroborate with development of inflammation and autoimmunity in thromboangiitis obliterans patients. Clin Exp Immunol 170: 28-35, 2012.

This work is licensed under a Creative Commons Attribution-NonCommercial-NoDerivatives 4.0 International (CC BY-NC-ND 4.0) License. 